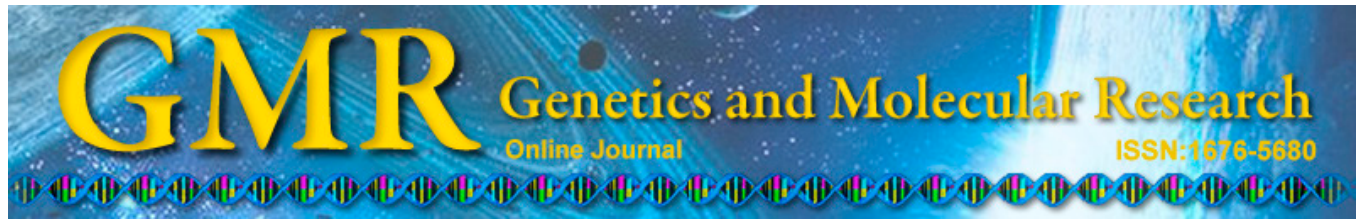

\title{
Low-level sequence variation in Toxoplasma gondii calcium-dependent protein kinases among different genotypes
}

\author{
J.L. Wang ${ }^{1}$, N.Z. Zhang ${ }^{1}$, S.Y. Huang ${ }^{1}$, Y. Xu ${ }^{1,2}$, R.A. Wang ${ }^{3}$ and X.Q. Zhu ${ }^{1}$ \\ ${ }^{1}$ State Key Laboratory of Veterinary Etiological Biology, \\ Key Laboratory of Veterinary Parasitology of Gansu Province, \\ Lanzhou Veterinary Research Institute, \\ Chinese Academy of Agricultural Sciences, Lanzhou, Gansu Province, China \\ ${ }^{2}$ College of Animal Science and Technology, Anhui Agricultural University, \\ Hefei, Anhui Province, China \\ ${ }^{3}$ College of Veterinary Medicine, South China Agricultural University, \\ Guangzhou, Guangdong Province, China \\ Corresponding author: S.Y. Huang \\ E-mail: siyang.huang@hotmail.com
}

Genet. Mol. Res. 14 (2): 4949-4956 (2015)

Received July 29, 2014

Accepted October 29, 2014

Published May 11, 2015

DOI http://dx.doi.org/10.4238/2015.May.11.28

\begin{abstract}
The causative agent of toxoplasmosis, Toxoplasma gondii, can infect virtually all nucleated cell types of warm-blooded animals. In this study, we examined the sequence variation in calciumdependent protein kinase $2(C D P K 2)$ genes among $13 \mathrm{~T}$. gondii strains from different hosts and geographical locations. The results showed that the lengths of the complete CDPK2 DNA and cDNA sequences were 3671-3673 and $2136 \mathrm{bp}$, respectively, and the sequence variation was $0-0.9 \%$ among different $T$. gondii strains. Phylogenetic analysis based on the CDPK2 gene sequences revealed that $T$. gondii strains
\end{abstract}


of the same genotypes were clustered in different clades. Further analysis of all the other $T$. gondii $C D P K$ genes in genotype I (GT1), II (ME49), or III (VEG) strains indicated the T. gondii CDPK gene family is quite conserved, with sequence variation ranging from 0 to $1.40 \%$. We concluded that $C D P K 2$ as well as all the other $C D P K$ genes in $T$. gondii cannot be used as proper markers for studying the variants of different $T$. gondii genotypes from different hosts and geographical locations, but their sequence conservation may be a useful feature promoting them as anti- $T$. gondii vaccine candidates in further studies.

Key words: Toxoplasma gondii; Calcium-dependent protein kinases; Sequence variation; Genotypes

\section{INTRODUCTION}

The apicomplexan Toxoplasma gondii is an obligate intracellular pathogen that can infect virtually all warm-blooded animals and humans, causing toxoplasmosis (Dubey, 2010; Zhou et al., 2011; Robert-Gangneux and Dardé, 2012). T. gondii infection is normally asymptomatic but can cause serious health problems in congenitally infected newborns and immune-compromised individuals (Luft and Remington, 1992; Boyer et al., 2005). Toxoplasmosis in animals, especially in pigs and goats, can cause infertility, spontaneous abortion, and neonatal malformations, which lead to serious economic impacts on livestock industries (Bhopale, 2003). Importantly, consumption of raw or undercooked meat containing $T$. gondii tissue cysts is the main source of human infection and thus raises public health concerns (Tenter et al., 2000).

Calcium-dependent protein kinases (CDPKs) are widely distributed, and found in many kinds of plants, ciliates, and apicomplexan parasites, but are absent in animals and humans (Harper and Harmon, 2005). CDPKs are, however, abundant in apicomplexans (Nagamune and Sibley, 2006). T. gondii, for example, was estimated to possess 12 CDPK isoforms (Billker et al., 2009), which have been shown to control diverse important biological functions (Lourido et al., 2011, 2012; McCoy et al., 2012; Morlon-Guyot et al., 2014). In

plants, $C D P K$ genes are highly conserved (Cheng et al., 2002), but little is known regarding the sequence variation of members of $C D P K$ family genes among different $T$. gondii strains. Therefore, the present study was designed to investigate sequence diversity in $C D P K$ genes among $T$. gondii strains from different genotypes.

\section{MATERIAL AND METHODS}

\section{T. gondii isolates}

A total of 13 T. gondii strains stemming from different hosts and geographic locations were used for analysis in this study (Table 1). All the T. gondii strains have been genotyped in previous studies and their genomic DNA was prepared as described previously (Zhou et al., 2009, 2010; Su et al., 2010; Huang et al., 2012). 
Table 1. Details of Toxoplasma gondii isolates used in this study.

\begin{tabular}{llll}
\hline Strain & Host & Geographical origin & Genotype \\
\hline RH & Human & France & Reference, Type I, ToxoDB \#10 \\
GT1 & Goat & United States & Reference, Type I, ToxoDB \#10 \\
PTG & Sheep & United States & Reference, Type II, ToxoDB \#1 \\
CTG & Cat & United States & Reference, Type III, ToxoDB \#2 \\
MAS & Human & France (Reference) & Reference ToxoDB \#17 \\
Prugniaud (PRU) & Human & France & Type II, ToxoDB \#1 \\
TgC7 & Cat & Guangzhou, Guangdong, China & ToxoDB \#9 \\
PYS & Pig & Panyu, Guangdong, China & ToxoDB \#9 \\
TgCgCa1 & Cougar & Canada & Reference, ToxoDB \#66 \\
TgCatBr5 & Cat & Brazil & Reference, ToxoDB \#19 \\
FOU & Human & France & Reference, ToxoDB \#6 \\
VEG & Human & United States & Reference, ToxoDB \#2 \\
ME49 & Sheep & United States & Type II, ToxoDB \#1 \\
\hline
\end{tabular}

\section{Polymerase chain reaction (PCR) amplification of CDPK2 genes}

The entire genomic sequence of the CDPK2 genes from individual T. gondii strains was amplified using the oligonucleotide primers FCDPK2 (forward primer: 5'-ATG CCG CTC AAG ACT TCC TGG CAT T-3') and RCDPK2 (reverse primer: 5'-TTA CCC CGT AGC GCG AGG CGT CAG ACT G-3'). The amplification reaction was carried out in a $25-\mu \mathrm{L}$ total volume, including 12.5 $\mu \mathrm{L}$ Premix Ex Taq (TaKaRa Bio, Otsu, Shiga, Japan), $0.2 \mu \mathrm{M}$ of each primer, and 100-200 ng template DNA. Amplification of DNA samples from individual strains was carried out in a thermocycler (Bio-Rad, Hercules, CA, USA) under the following conditions: denaturation at $94^{\circ} \mathrm{C}$ for $10 \mathrm{~min}$ (initial denaturation), followed by 35 cycles of $94^{\circ} \mathrm{C}$ for $50 \mathrm{~s}$ (denaturation), $67^{\circ} \mathrm{C}$ for $40 \mathrm{~s}$ (annealing), $72^{\circ} \mathrm{C}$ for $3 \mathrm{~min}$ and $40 \mathrm{~s}$ (extension), and a final extension step at $72^{\circ} \mathrm{C}$ for $10 \mathrm{~min}$. The successful PCR amplifications were confirmed by electrophoresis on a $1 \%(\mathrm{w} / \mathrm{v})$ agarose gel, and stained with GoldenView ${ }^{\mathrm{TM}}$ and the DL 5000 marker (TaKaRa) to estimate the sizes of the CDPK2 PCR products.

\section{DNA sequencing}

PCR products were purified using the PCR-Preps DNA Purification System (Promega, Madison, WI, USA) according to manufacturer recommendations, and then ligated to the pMD18$\mathrm{T}$ vector (TaKaRa). The recombinant vectors were transformed into JM109 competent cells (Promega). Positive recombinant vectors were identified by bacterial PCR amplification directly with the same primers described previously, and sent to Sangon (Shanghai, China) for sequencing.

\section{Sequence analysis and phylogenetic reconstruction}

The CDPK gene sequences of the VEG, FOU, ME49, and GT1 strains were obtained from the ToxoDB database (http://toxodb.org). All the obtained T. gondii CDPK sequences were aligned using the computer program Clustal X 1.81 (Thompson et al., 1997) and then the sequence variation was determined among the examined T. gondii strains. Phylogenetic reconstructions based on the complete $C D P K 2$ gene sequences of different $T$. gondii strains were performed using maximum parsimony (MP), and neighbor joining (NJ) with the outgroup using the corresponding sequence from Neospora caninum (GenBank accession No. FR823391.1). MP and NJ analyses were performed using PAUP* 4.0 with indels treated as missing 
character states (Swofford, 2002). A total of 1000 random addition searches using tree bisectionreconnection branch swapping were performed for each MP and NJ analysis. Bootstrap probability was calculated from 1000 bootstrap replicates with 10 random additions per replicate in PAUP. The nucleotide composition, transition, tranversion, and the genetic distance calculations were performed using the MegAlign program in the DNA Star Version 5.0 software (Xu et al., 2014).

\section{RESULT AND DISCUSSION}

The obtained amplicons of the CDPK2 genes were determined to be approximately $3600 \mathrm{bp}$ in length by agarose gel electrophoresis for all $10 \mathrm{~T}$. gondii strains. The sequence results showed that the complete genomic sequence of the CDPK2 gene was $3671 \mathrm{bp}$ in length for the strains $\mathrm{TgC7}$ and PYS, $3673 \mathrm{bp}$ in length for MAS and $\mathrm{TgCgCa} 1$, and $3762 \mathrm{bp}$ for the others. Comparison of the CDPK2 genomic sequences using the Gene Structure Display Server (GSDS) (Guo et al., 2007) identified that four introns and five exons were distributed across the $C D P K 2$ gene. A total of 52 variable nucleotide positions containing 46 nucleotide substitutions, one single nucleotide polymorphism, and five nucleotide losses were identified in the CDPK2 gene sequences, with intra-specific variations of $1.42 \%(52 / 3673)$ for genomic DNA sequences and $0.66 \%(14 / 2136)$ for cDNA sequences. Fourteen nucleotide substitutions were identified in the expressed regions that were predicted to result in three amino acid substitutions (Table 2). The transitions and transversions in the entire genomic sequences and in the coding region of $T$. gondii $C D P K 2$ genes are shown in Table 3, which indicated that the intra-specific nucleotide variations occurred primarily in introns.

The phylogenetic reconstruction based on CDPK2 sequences from 13 T. gondii strains showed that the same genotypes were not clustered in a clade (Figure 1), which was similar to previous results from our group of phylogenetic reconstruction using PLP1 (Yan et al., 2011), ROP7 (Zhou et al., 2012), eIF4A (Chen et al., 2014), MIC13 (Ren et al., 2011), and ROP38 (Xu et al., 2014). The results from this analysis suggested that the T. gondii CDPK2 gene would not be an ideal genetic marker for intra-species phylogenetic analysis to identify different genotypes of $T$. gondii isolates from different hosts and geographical locations because of its low variation.

Further analysis of the other $T$. gondii $C D P K$ genes based on the genomic sequences available in the ToxoDB database showed similar low variation of $C D P K$ genes among $T$. gondii genotypes I, II, and III in general, with the highest genetic distance found in $\operatorname{TgCDPK6}(1.4 \%)$ and the lowest in $T g C D P K 14$ and $4 A$ genes $(0 \%)$ (Table 3 ). The low variation of $C D P K$ genes suggested conserved functionality for each $C D P K$ gene in different $T$. gondii strains as well. In previous studies, $T g C D P K 1, T g C D P K 3$, and $T g C D P K 7$ were shown to participate in parasite motility, such as host-cell invasion and egress, and parasite division and growth (Lourido et al., 2011, 2012; McCoy et al., 2012; Morlon-Guyot et al., 2014). The important functions and the low variation of CDPKs in different $T$. gondii strains imply that they may be considered promising vaccine candidates, as the protective immunity induced by $T$. gondii CDPK 3 revealed in our previous study showed the suitability of this protein family for development of new vaccines against $T$. gondii parasite infection (Zhang et al., 2013).

In conclusion, the present study revealed the low variability in CDPK2 gene sequences among $T$. gondii isolates from different geographical regions and hosts and further investigated the sequence variation in all $C D P K$ s among different $T$. gondii genotypes. This analysis showed that the $C D P K$ gene family in $T$. gondii is very conserved, which suggests that $T$. gondii $C D P K$ genes may be useful as candidate vaccines against toxoplasmosis. 


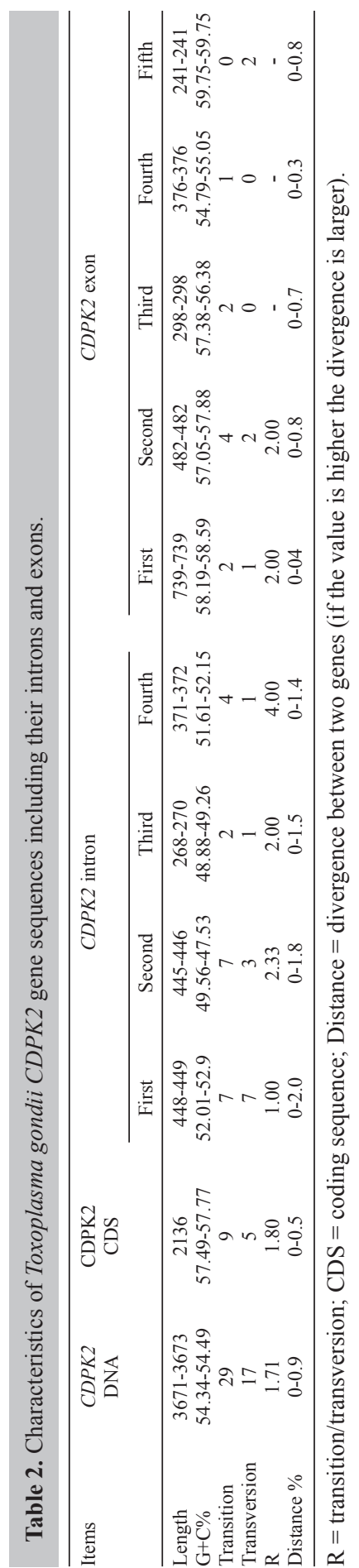


J.L. Wang et al.

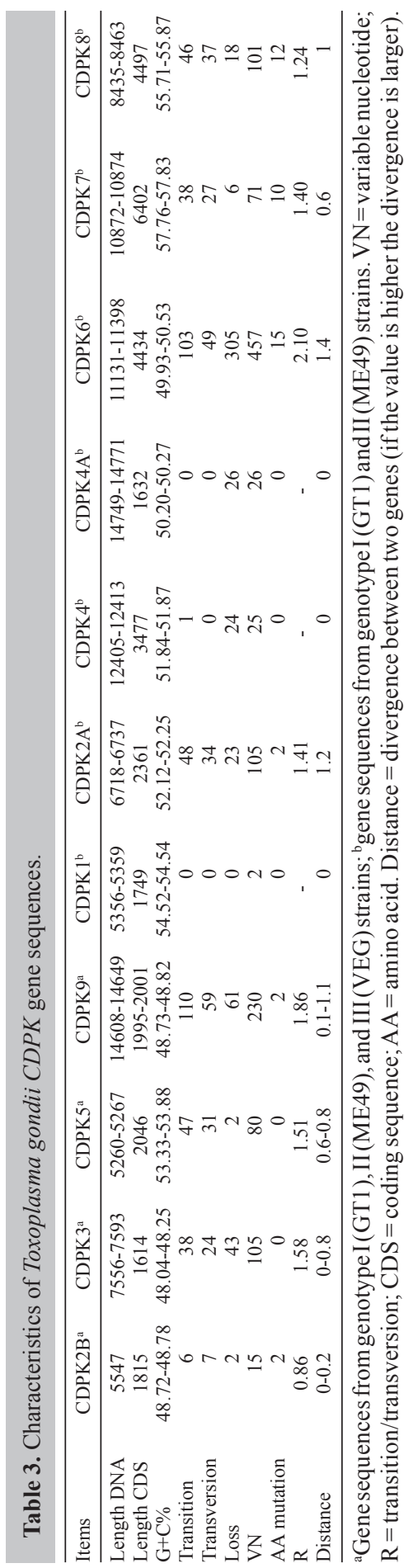




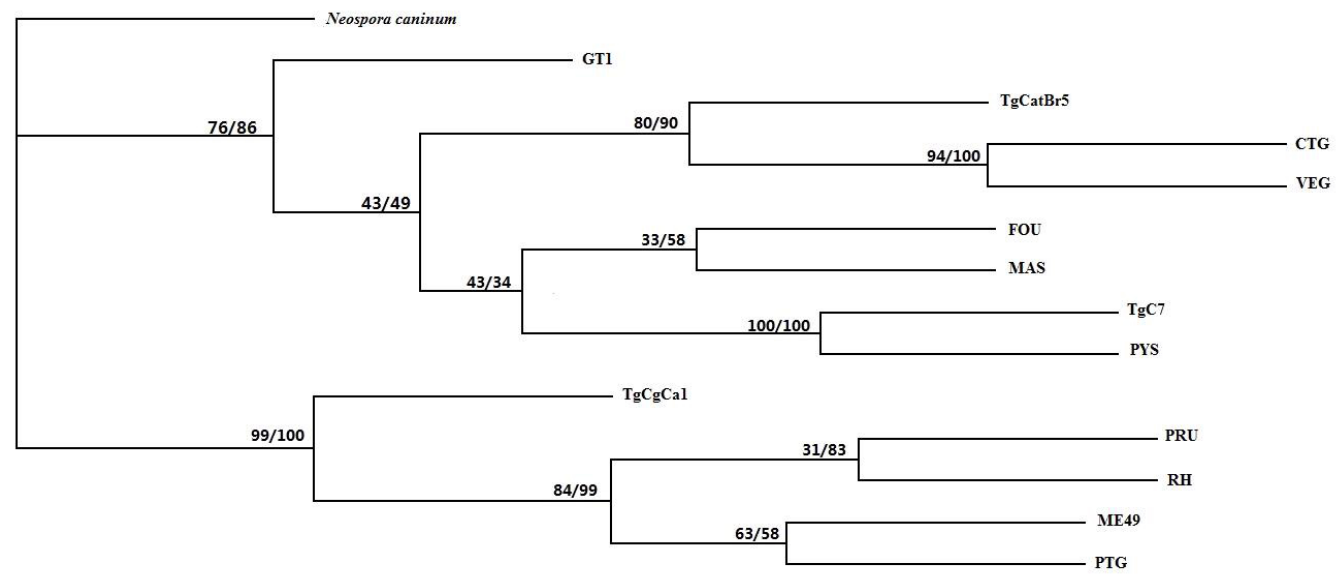

Figure 1. Phylogenetic relationships of Toxoplasma gondii isolates from different hosts and geographical locations. Relationships were constructed by maximum parsimony (MP) and neighbor-joining (NJ) analyses based on the TgCDPK2 gene sequences using Neospora caninum (GenBank accession No. FR823391.1) as the outgroup. Numbers of MP/NJ bootstrap values (\%) are indicated along the branches.

\section{ACKNOWLEDGMENTS}

Research supported by the National Natural Science Foundation of China (Grant \#31472184, \#31101812, \#31230073, and \#31172316), and the Science Fund for Creative Research Groups of Gansu Province (Grant \#1210RJIA006).

\section{REFERENCES}

Bhopale GM (2003). Development of a vaccine for toxoplasmosis: current status. Microbes Infect. 5: 457-462.

Billker O, Lourido S and Sibley LD (2009). Calcium-dependent signaling and kinases in apicomplexan parasites. Cell Host Microbe 5: 612-622.

Boyer KM, Holfels E, Roizen N, Swisher C, et al. (2005). Risk factors for Toxoplasma gondii infection in mothers of infants with congenital toxoplasmosis: Implications for prenatal management and screening. Am. J. Obstet. Gynecol. 192: 564-571.

Chen J, Fang SF, Zhou DH, Li ZY, et al. (2014). Sequence variation in the Toxoplasma gondii eIF4A gene among strains from different hosts and geographical locations. Genet. Mol. Res. 13: 3356-3361.

Cheng SH, Willmann MR, Chen HC and Sheen J (2002). Calcium signaling through protein kinases. The Arabidopsis calcium-dependent protein kinase gene family. Plant Physiol. 129: 469-485.

Dubey JP (2010). Toxoplasmosis of animals and humans. 2nd edn. CRC Press, Boca Raton.

Guo AY, Zhu QH, Chen X and Luo JC (2007). GSDS: a gene structure display server. Yi Chuan 29: 1023-1026.

Harper JF and Harmon A (2005). Plants, symbiosis and parasites: a calcium signalling connection. Nat. Rev. Mol. Cell Biol. 6: 555-566.

Huang SY, Cong W, Zhou P, Zhou DH, et al. (2012). First report of genotyping of Toxoplasma gondii isolates from wild birds in China. J. Parasitol. 98: 681-682.

Lourido S, Shuman J, Zhang C, Shokat KM, et al. (2011). Calcium-dependent protein kinase 1 is an essential regulator of exocytosis in Toxoplasma. Nature 465: 359-362.

Lourido S, Tang K and Sibley LD (2012). Distinct signalling pathways control Toxoplasma egress and host-cell invasion. EMBO J. 31: 4524-4534.

Luft BJ and Remington JS (1992). Toxoplasmic encephalitis in AIDS. Clin. Infect. Dis. 15: 211-222.

McCoy JM, Whitehead L, van Dooren GG and Tonkin CJ (2012). TgCDPK3 regulates calcium-dependent egress of Toxoplasma gondii from host cells. PLoS Pathog. 8: e1003066. 
Morlon-Guyot J, Berry L, Chen CT, Gubbels MJ, et al. (2014). The Toxoplasma gondii calcium-dependent protein kinase 7 is involved in early steps of parasite division and is crucial for parasite survival. Cell Microbiol. 16: 95-114

Nagamune K and Sibley LD (2006). Comparative genomic and phylogenetic analyses of calcium ATPases and calciumregulated proteins in the apicomplexa. Mol. Biol. Evol. 23: 1613-1627.

Ren D, Zhou DH, Xu MJ, Zhou Y, et al. (2011). Sequence variation in Toxoplasma gondii MIC13 gene among isolates from different hosts and geographical locations. Afr. J. Microbiol. Res. 6: 3265-3269.

Robert-Gangneux F and Dardé ML (2012). Epidemiology of and diagnostic strategies for toxoplasmosis. Clin. Microbiol. Rev. 2: 264-296.

Su C, Shwab EK, Zhou P, Zhu XQ, et al. (2010). Moving towards an integrated approach to molecular detection and identification of Toxoplasma gondii. Parasitology 137: 1-11.

Swofford DL (2002). PAUP: phylogenetic analysis using parsimony (*and other methods). Version 4.0, Sinauer Associates, Sunderland.

Tenter AM, Heckeroth AR and Weiss LM (2000). Toxoplasma gondii: from animals to humans. Int. J. Parasitol. 30 : 1217-1258.

Thompson JD, Gibson TJ, Plewniak F, Jeanmougin F, et al. (1997). The CLUSTAL X windows interface: flexible strategies for multiple sequence alignment aided by quality analysis tools. Nucleic Acids Res. 25: 4876-4882.

Xu Y, Zhang NZ, Chen J, Zhu XQ, et al. (2014). Toxoplasma gondii rhoptry protein 38 gene: Sequence variation among isolates from different hosts and geographical locations. Genet. Mol. Res. 13: 4839-4844.

Yan HK, Song HQ, Zhou Y, Ren D, et al. (2011). Sequence variation in perforin-like protein 1 gene among six Toxoplasma gondii strains. J. Anim. Vet. Adv. 10: 2244-2247.

Zhang NZ, Huang SY, Zhou DH, Chen J, et al. (2013). Protective immunity against Toxoplasma gondii induced by DNA immunization with the gene encoding a novel vaccine candidate: calcium-dependent protein kinase 3. BMC Infect. Dis. 13: 512 .

Zhou P, Zhang H, Lin RQ, Zhang DL, et al. (2009). Genetic characterization of Toxoplasma gondii isolates from China. Parasitol. Int. 58: 193-195.

Zhou P, Nie H, Zhang LX, Wang HY, et al. (2010). Genetic characterization of Toxoplasma gondii isolates from pigs in China. J. Parasitol. 96: 1027-1029.

Zhou P, Chen Z, Lin RQ, Zhu XQ, et al. (2011). Toxoplasma gondii infection in humans in China. Parasit. Vectors 4: 165.

Zhou Y, Lu P, Xu MJ, Ren D, et al. (2012). Sequence variation in TgROP7 gene among Toxoplasma gondii isolates from different hosts and geographical regions. Afr. J. Biotechnol. 11: 6658-6661. 